\title{
ORIGINAL ARTICLE \\ The treatment of mild cervical spondylotic myelopathy with increased signal intensity on T2-weighted magnetic resonance imaging
}

\author{
FN Li ${ }^{1,2,5}, \mathrm{ZH} \mathrm{Li}^{3}{ }^{3,5}, \mathrm{X} \mathrm{Huang}^{2}, \mathrm{SZ} \mathrm{Yu}{ }^{4}, \mathrm{~F} \mathrm{Zhang}{ }^{2}, \mathrm{Z} \mathrm{Chen}^{2}, \mathrm{HX} \mathrm{Shen}^{2}, \mathrm{~B} \mathrm{Cai}^{2}$ and TS Hou ${ }^{2}$
}

Study design: A retrospective comparative study.

Objectives: To compare clinical outcomes of surgery or non-operated treatment for mild cervical spondylotic myelopathy (CSM) patients with intramedullary increased signal intensity (ISI) on T2-weighted imaging (T2WI) of magnetic resonance imaging (MRI), related factors that may affect prognosis were explored.

Methods: Data from 91 patients treated from July 2008 to June 2011 were retrospectively analyzed. The Japanese Orthopedic Association (JOA) recovery ratio was used to compare outcomes of surgery and non-operated treatment. Correlation and multiple linear regression analyses were performed between JOA recovery ratio and age, disease course, segmental lordosis, total range of motion (ROM), segmental ROM, local slip, extent of spinal cord compression or ISI type.

Results: Patients were divided into two groups by therapy methods: Group A ( $n=53,33$ males, age 36-68 years) underwent anterior cervical decompression and fusion surgery, average follow-up 30.68 \pm 8.19 months; Group B ( $n=38,14$ males, age 28-76 years) received non-operated treatment, average follow-up 34.08 \pm 9.05 months. There were no significant differences in clinical outcomes between the two groups. There were significant correlations between JOA recovery ratio and clinical course $(P<0.01)$ or segmental lordosis $(P<0.01)$. Patients with shorter disease course and larger segmental lordosis have better clinical outcomes as shown by multiple linear regression analysis.

Conclusion: For mild CSM patients with ISI on T2WI-MRI, there are no significant differences in clinical outcome between surgery and non-operated treatment during the short-term follow-up. Patients with shorter disease course and larger segmental lordosis have better clinical outcomes than those with longer course and segmental kyphosis.

Spinal Cord (2014) 52, 348-353; doi:10.1038/sc.2014.11; published online 18 February 2014

Keywords: cervical spondylotic myelopathy; increased signal intensity; magnetic resonance imaging; T2-weighted imaging.

\section{INTRODUCTION}

Cervical spondylotic myelopathy (CSM) is described by symptoms and signs caused by stimulation and compression of the spinal cord due to cervical intervertebral disk degeneration diseases and secondary changes. Because of occult onset and continuous aggravation, severe clinical symptoms and signs usually appear at late-stage disease, leading to poor prognosis and seriously affecting the quality of life. ${ }^{1}$ As CSM is characterized by chronic compression of the spinal cord in the cervical spine, this increased pressure causes ischemic injury and even the degeneration of spinal cord, and these lesions show increased signal intensity (ISI) on T2-weighted imaging (T2WI) of magnetic resonance imaging (MRI). ${ }^{2}$ It has been reported by meta-analysis that the prognosis of CSM patients with intramedullary ISI is poorer than those without intramedullary ISI. $^{3}$ For mild-to-moderate CSM patients, there is no significant difference between surgery and conservative treatment as reported. ${ }^{4-6}$

However, there is no necessary correlation of spinal cord compression and intramedullary signal intensity with clinical symptoms. Sometimes, we may meet such patients whose clinical symptoms are relatively mild, although severe spinal cord compression is shown by MRI even with intramedullary ISI on T2WI. For these patients, it is more difficult to determine the disease progression and surgery time, and because surgical intervention is later, postoperative neurological functional recovery might be poorer.

The aim of this research was to compare the clinical outcomes of mild CSM patients with intramedullary ISI on T2WI who received surgery or non-operated treatment to find out whether a surgical procedure is needed to proceed for these patients. We collected data from mild CSM patients who met the inclusion requirements, in order to explore the related factors that may affect prognosis.

\section{MATERIALS AND METHODS}

\section{Patient population}

Inclusion criteria: single level CSM patients with intramedullary ISI on T2WI and Japanese Orthopedic Association (JOA) $\geqslant 13$. Exclusion criteria: multilevel CSM; history of trauma; CSM combined with cervical spondylotic radiculopathy; CSM combined with ossification of posterior longitudinal ligament. Outpatients and inpatients with CSM treated in our hospital from

${ }^{1}$ Department of Orthopedics, Third Affiliated Hospital of PLA Second Military Medical University, Shanghai, People's Republic of China; ${ }^{2}$ Department of Orthopedics, Changhai Hospital, Second Military Medical University, Shanghai, People's Republic of China; ${ }^{3}$ Department of Orthopedics, First Affiliated Hospital of PLA General Hospital, Beijing, People's Republic of China and “Department of Orthopedics, Tenth People's Hospital of TongJi University, Shanghai, People's Republic of China

5These two authors contributed equally to this work.

Correspondence: Professor HX Shen or Dr B Cai, Department of Orthopedics, Changhai Hospital, Second Military Medical University, 168 Changhai Road, Shanghai 200433, People's Republic of China.

E-mail: shenhxspine@gmail.com or 52685590@qq.com

Received 28 September 2013; revised 8 January 2014; accepted 14 January 2014; published online 18 February 2014 
July 2008 to June 2011 were retrospectively analyzed. A total of 91 cases qualified for the inclusion criteria. The data were divided into two groups according to therapy method: Group A underwent anterior cervical decompression and fusion surgery, Group B refused surgery because of poor economic conditions and received non-operated treatment.

\section{Treatment methods}

Surgery method: all patients received anterior cervical decompression and fusion surgery. X-ray perspective was used to determine operation section. Patients were instructed to wear a neck collar for 6 weeks for protection and followed up at 6-weeks, 3, 6, 12, 18 and 24 months postoperatively.

Non-operated treatment included the following: oral drugs (for neuronutrition, diminished inflammation and pain relief), traction, acupuncture, physiotherapy, and so on.

\section{Collected data and outcome assessment}

Clinical outcome, including prior-treatment and post-treatment JOA score, Neck Disability Index (NDI) score, JOA recovery ratio $=$ (post-treatment JOA score - prior-treatment JOA score $) /(17$ - prior-treatment JOA score $)$ $\times 100 \%$.

Imaging, including range of motion ( $\mathrm{ROM}$ ) of $\mathrm{C} 2-\mathrm{C} 7$ : the curve value is characterized as the angle between the link line of inferior edges of C2 and C7 vertebrae. The value is chosen by subtracting the flexion angle from the extension angle; if the flexion angle was kyphosis, a negative value results (Figure 1). Segmental lordosis: the angle between the superior and inferior edges of connected vertebral bodies of the most compressed spinal cord segment is measured on MRI, with negative value result with kyphosis (Figure 2). Extent of spinal cord compression: the value measured as sagittal diameter of the most compressed spinal cord segment/sagittal diameter of the C1 segment; smaller values indicate more severe compression. We also measured presence or absence of a local slip of $2 \mathrm{~mm}$ or more and ROM at the maximum compression segment on MRI as Oshima described. ${ }^{6}$ On the basis of the ISI characteristics, we categorize ISI as fuzzy type or sharp type. ${ }^{7}$ All radiological outcomes was evaluated by two independent examiners.

\section{Statistical analysis}

All data analyses were conducted using SPSS 19 software (SPSS Inc., Chicago, IL, USA). The quantitative data, such as age, JOA scores and NDI scores are presented as the mean \pm s.d. Intergroup comparisons were made by $t$-test; Pearson correlation analysis was performed between JOA recovery ratio and ages, disease course, segmental lordosis, ROM (C2-7), segmental ROM or extent of spinal cord compression. Spearman correlation analysis was performed between JOA recovery ratio and therapy methods, local slip, gender or ISI type. Statistical analysis of factors influencing the treatment clinical curative effect was performed with multiple linear regression (differences were considered statistically significant when $P<0.05$ ).

\section{RESULTS}

\section{Patients data}

A total of 91 cases were included in the study. Group A included 53 cases ( 33 males, 20 females), the average age was $50.36 \pm 8.13$ years, and average follow-up was $30.68 \pm 8.19$ months. Group B included 38 cases (14 males, 24 females), the average age was $52.95 \pm 11.64$ years, and average follow-up was $34.08 \pm 9.05$ months. There was no significant difference in age or duration of follow-up between the two groups. Table 1 summarizes the demographics of the patients, imaging results and clinical therapy effects of the two groups. As compared with the non-operated treatment group, no obvious advantage was shown in the JOA recovery ratio and the NDI score of surgery groups. There was no significant difference in clinical effects between the two groups. Typical patients of both the groups are shown in Figures 3 and 4.

\section{Pearson and Spearman correlation analysis results}

There is significant correlation between JOA recovery ratio and disease course $(P<0.01)$ or segmental lordosis $(P<0.01)$, whereas no significant correlation between JOA recovery ratio and ages, clinical course, segmental lordosis, total ROM (C2-7), segmental ROM, local slip, extent of spinal cord compression or ISI type (Table 2).

\section{Multiple linear regression analysis results}

The significant correlative index, disease course, segmental lordosis and JOA recovery ratio were analyzed by multiple linear regression analysis. Regression formula: JOA recovery ratio $=0.495-0.011 \times$ disease course $+0.097 \times$ segmental lordosis.

Two statistical significant predictive index correlating with JOA recovery ratio were disease course $(P<0.01)$ and segmental lordosis $(P<0.01)$. Correlation coefficient of regression formula $R=0.888$, determination coefficient $R^{2}=0.785$, hypothesis testing $F=297.225$
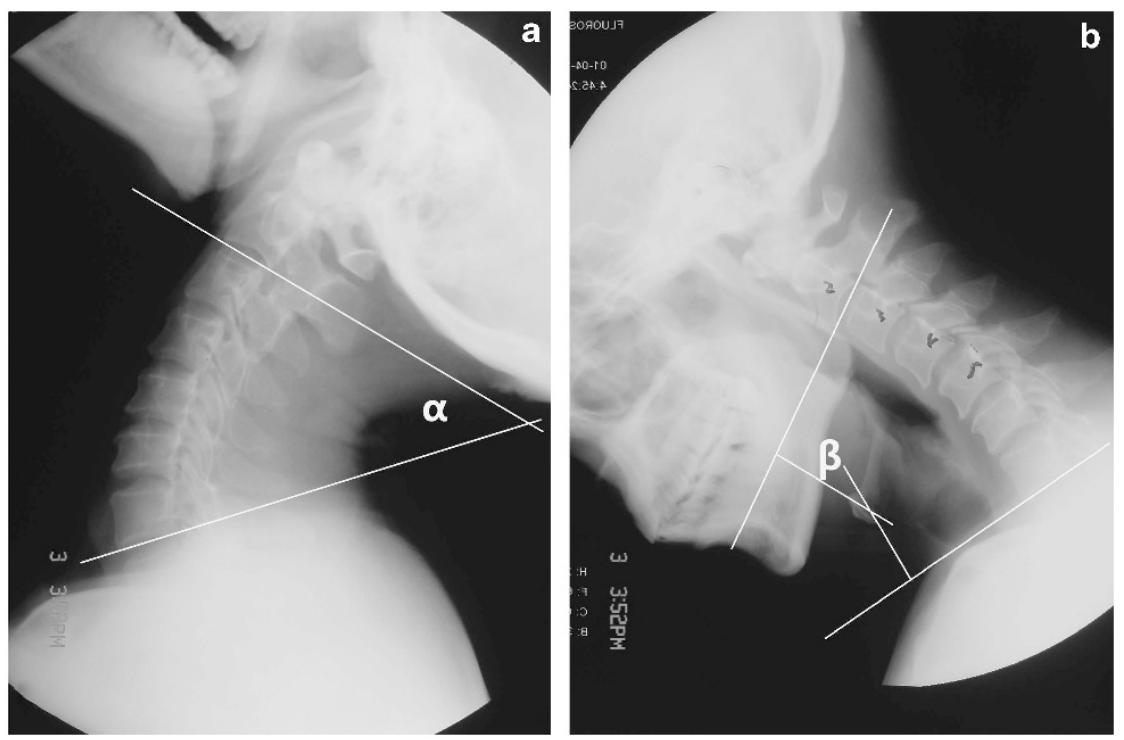

Figure 1 ROM of C2-C7: curve value is characterized as the angle between the link line of the inferior edges of C2 and C7 vertebrae; negative values result with kyphosis. 

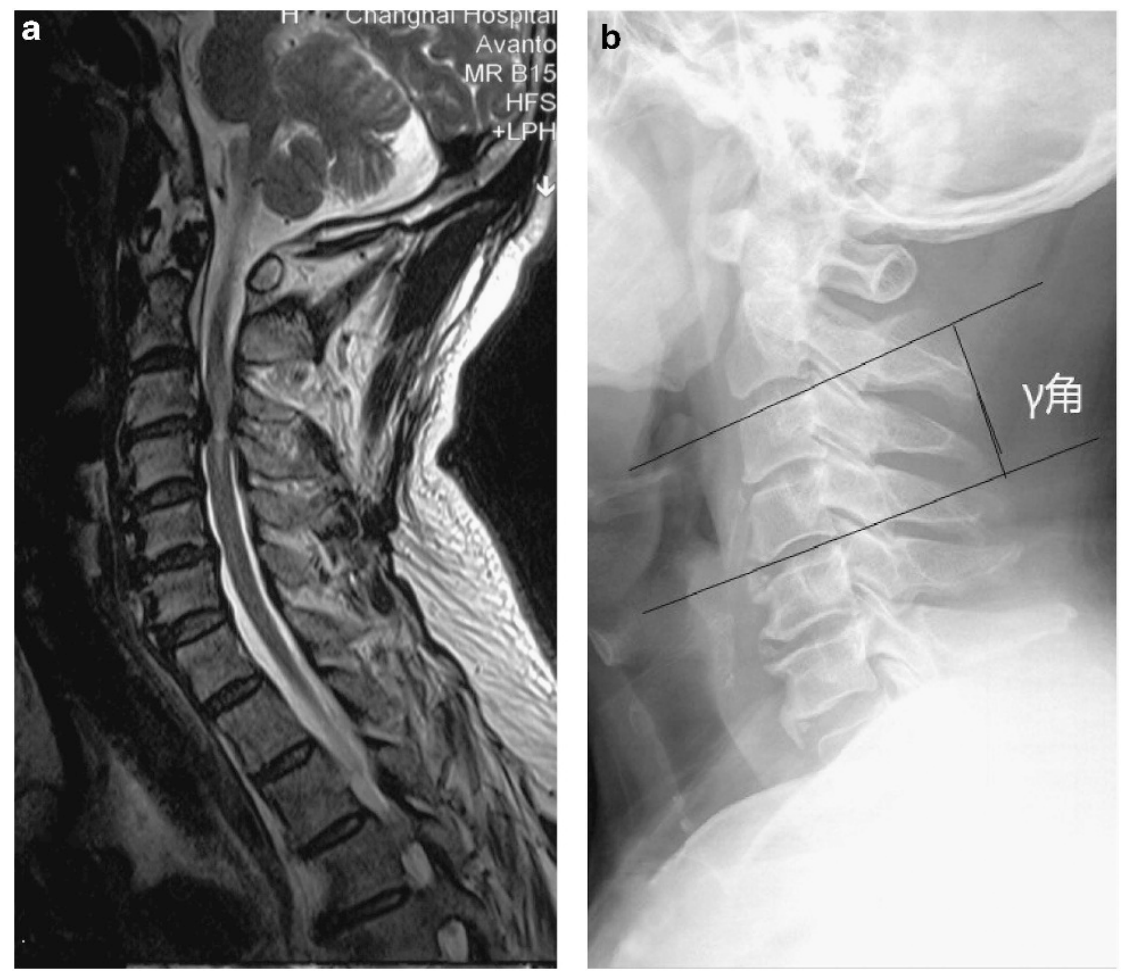

Figure 2 Segmental lordosis: the angle between the superior and inferior edges of connected vertebral bodies of the most compressed spinal cord segment measured on MRI; negative value indicate kyphosis.

Table 1 Basic information, imaging results, and clinical therapy effects of surgery group and non-operated treatment group

\begin{tabular}{lccc}
\hline & $\begin{array}{c}\text { Surgery group } \\
(\mathrm{n}=53)\end{array}$ & $\begin{array}{c}\text { Non-operated group } \\
(\mathrm{n}=38)\end{array}$ & $\begin{array}{c}\mathrm{P} \text { - } \\
\text { value }\end{array}$ \\
\hline Age (year) & $50.36 \pm 8.13$ & $52.95 \pm 11.64$ & 0.056 \\
Gender (male:female) & $33: 20$ & $14: 24$ & 0.863 \\
Disease course (month) & $4.69 \pm 3.28$ & $5.97 \pm 5.08$ & 0.087 \\
Duration of follow-up & $30.68 \pm 8.19$ & $34.08 \pm 9.05$ & 0.351 \\
(month) & & & \\
Total ROM (C2-7) ( $\left.{ }^{\circ}\right)$ & $54.52 \pm 6.93$ & $52.36 \pm 6.73$ & 0.745 \\
Segmental ROM ( $\left.{ }^{\circ}\right)$ & $12.04 \pm 2.07$ & $11.96 \pm 2.21$ & 0.676 \\
Segmental lordosis ( $\left.{ }^{\circ}\right)$ & $0.76 \pm 2.29$ & $0.23 \pm 2.51$ & 0.585 \\
Extent of spinal cord & $53.97 \pm 6.57$ & $50.46 \pm 5.27$ & 0.312 \\
compression (\%) & & & \\
Local slip (Yes:No) & $5: 48$ & $2: 36$ & 0.140 \\
ISI type (fuzzy:sharp) & $31: 22$ & $19: 19$ & 0.296 \\
Prior-treatment JOA score & $14.23 \pm 1.07$ & $14.37 \pm 0.97$ & 0.365 \\
Post-treatment JOA score & $15.60 \pm 0.91$ & $15.45 \pm 0.92$ & 0.891 \\
JOA recovery ratio (\%) & $52.83 \pm 27.44$ & $43.86 \pm 29.55$ & 0.646 \\
Prior-treatment NDI (\%) & $21.15 \pm 4.98$ & $20.82 \pm 4.24$ & 0.303 \\
Post-treatment NDI (\%) & $18.03 \pm 4.76$ & $18.73 \pm 4.54$ & 0.991 \\
\hline
\end{tabular}

Values shown are mean \pm s.d.

$(P<0.01)$, specified the formula with statistical significance. As shown in the linear regression formula, patients with shorter disease course and larger segmental lordosis have better clinical outcomes.

\section{DISCUSSION}

Aiming at the clinical therapy of mild CSM patients with intramedullary ISI on T2WI of MRI, we collected the data of patients undergoing surgery and non-operated treatment in our hospital. No significant differences in clinical outcomes between the two groups were shown during the follow-up. The potential factors linked with clinical outcomes were further analyzed by using multiple linear regression analysis. It turned out that the disease course and segmental lordosis was significantly correlated with JOA recovery ratio, specifying that patients with shorter disease course and larger segmental lordosis have better clinical outcomes. Because of the shortterm follow-up, the results may not withstand the test of time. All patients in the study, even receiving non-operated treatment, had a stable clinical course, which may be associated with the short-term follow-up. However, we think that it is reasonable to conclude that for most patients, there is a relatively low risk for spinal cord injury in the 2-4-year follow-up period. Therefore, there does not appear to be an urgent need for surgical treatment.

The relationship between intramedullary ISI on T2WI and surgical prognosis has always been controversial with three major viewpoints: (1) Previous research suggests that ISI means different extent of pathological changes at segmental spinal cord, even receive surgery, their prognosis are usually poor. ${ }^{2,8}$ (2) There is no correlation between ISI and severity of CSM as well as surgical prognosis; ; 10 some researchers reported that ISI correlated with surgical prognosis, but no significant difference has been showed by statistical analysis. $^{11-13}$ (3) Another viewpoint suggested is that the intramedullary ISI change is a gradual progress. At the early stage, ISI reflects edema of spinal cord; at the mid-stage, it reflects necrosis of gray matter cells; and at the late stage, it reflects formation of spinal cord cavity. For early stage, intramedullary ISI may decrease to an approximately normal level by surgical decompression. Necrosis and cavity at the mid-late stage are irreversible pathological changes, which may explain why the clinical outcome of surgery is poor. ${ }^{14-17}$ As shown by our previous meta-analysis, clinical outcomes of patients with intramedullary ISI on T2WI of MRI are poor. ${ }^{3}$ 

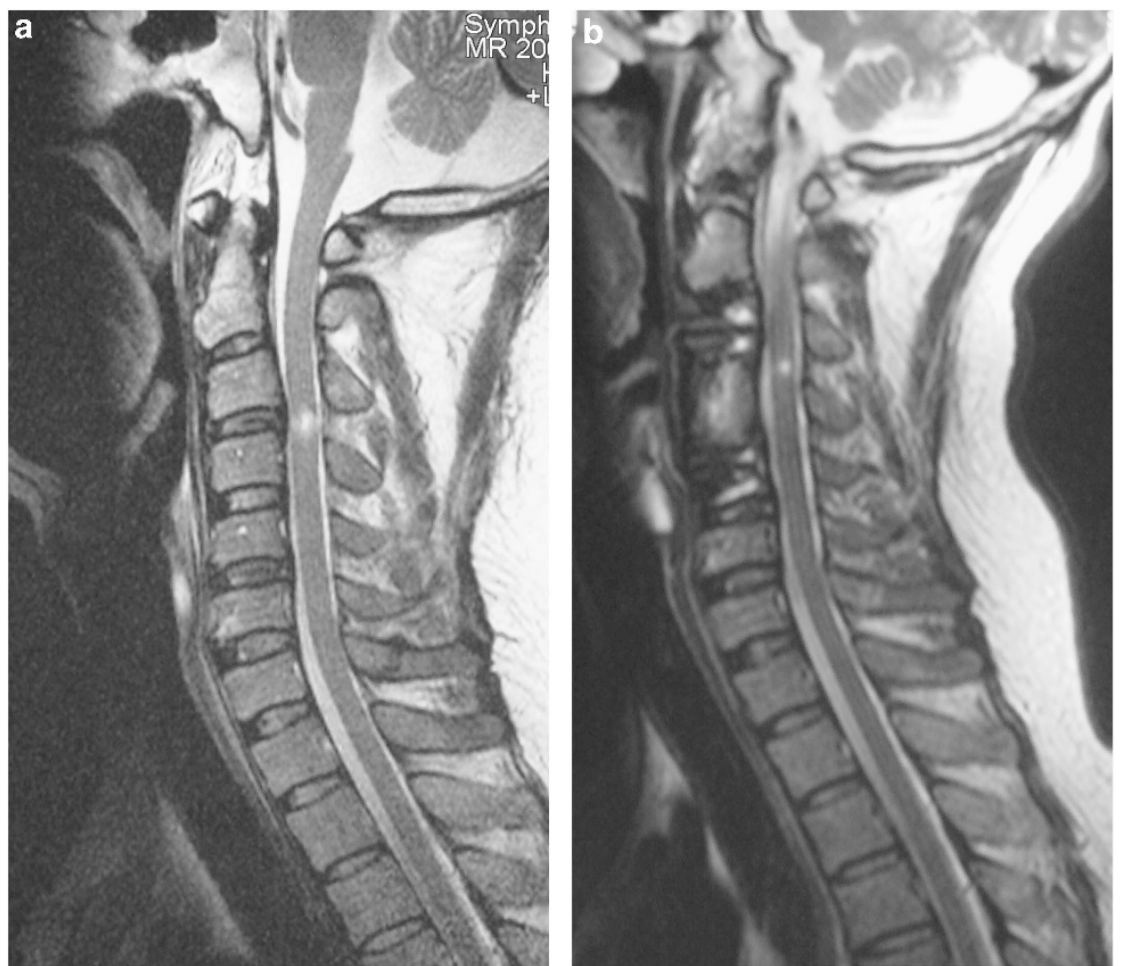

Figure 3 A 38-year-old male patient presented with a 3-month history of numbness in both hands which had become aggravated in the last month. This was accompanied by a month history of unsteady gait. Preoperative MRI image (a) shows intramedullary ISI behind C3-4 intervertebral disk. He underwent anterior cervical decompression and fusion surgery. Postoperative MRI images (b) three months after surgery shows the area of ISI decreased. His JOA and NDI score changed from 14 to 16 and 24 to 18 , respectively.
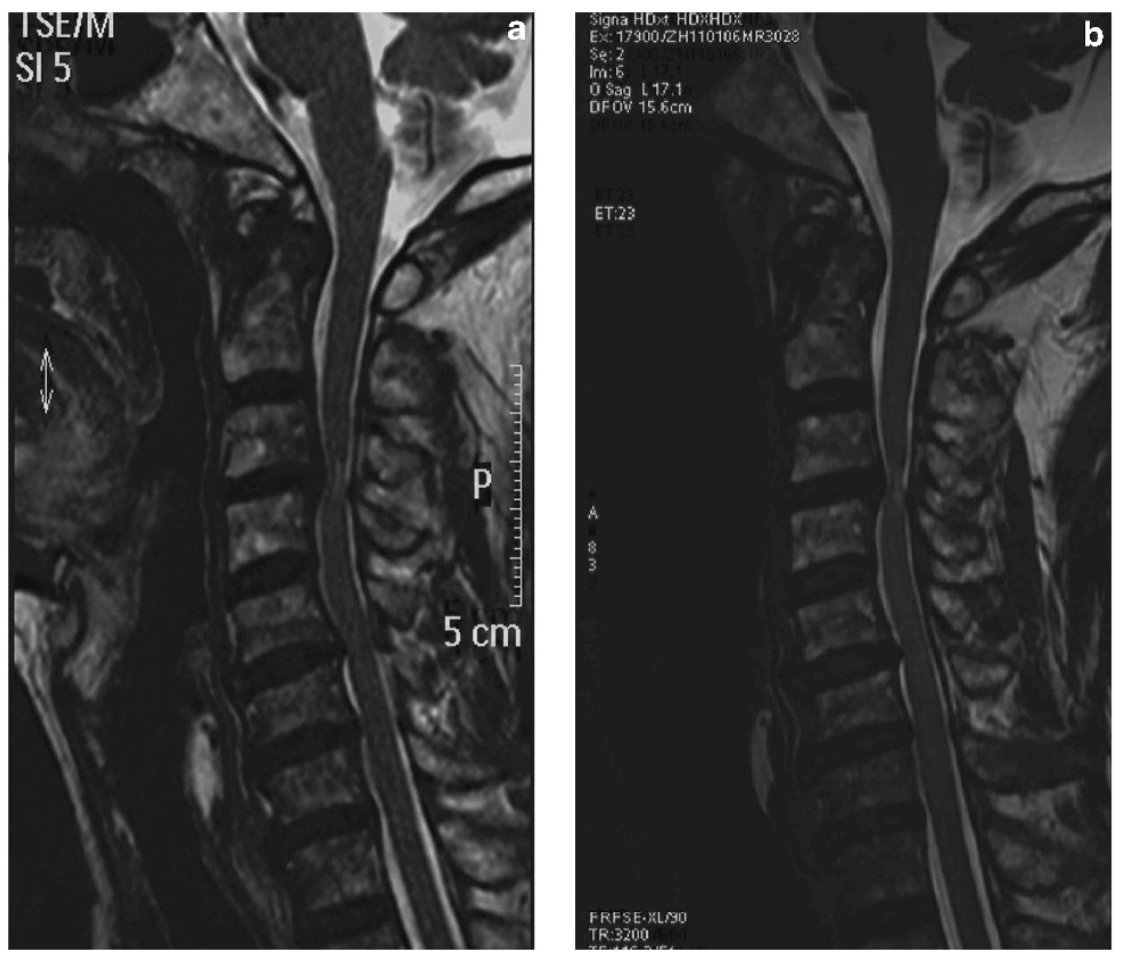

Figure 4 A 63-year-old male patient presented with a two-month history of limb weakness accompanied by unsteady gait. Prior-treatment MRI image (a) shows intramedullary ISI behind C3-4 intervertebral disk. He refused surgery because of fear of operation. (b) shows MRI image two years after non-operated treatment, the intramedullary ISI did not have much difference compared with before. His JOA and NDI score changed from 14 to 15 and 23 to 20 , respectively. 
Table 2 Correlation analysis results between JOA recovery ratio and other factors

\begin{tabular}{lcc}
\hline & Correlation coefficient- $\gamma$ & P-value \\
\hline Therapy methods & -0.159 & 0.133 \\
Gender & -0.126 & 0.236 \\
Age & -0.182 & 0.084 \\
Clinical course & -0.515 & $0.000^{*}$ \\
Segmental lordosis & 0.877 & $0.000^{*}$ \\
Total ROM (C2-7) & -0.093 & 0.382 \\
Segmental ROM & -0.016 & 0.880 \\
Local slip & 0.029 & 0.785 \\
Extent of spinal cord Compression (\%) & 0.128 & 0.227 \\
ISI type & -0.197 & 0.061 \\
\hline$* 0<0.05$. &
\end{tabular}

${ }^{*} P<0.05$.

Vedantam et al. ${ }^{18}$ reviewed the literature of ISI, and found that multisegmental ISI and sharp, intense ISI are associated with poorer surgical outcome and the regression of ISI postoperatively correlates with better functional outcomes. However, in our study, no significant differences in ISI type were shown between the two groups, and ISI type was not associated with JOA recovery ratio.

For the treatment of mild CSM, most researchers suggest there is no significant difference between conservative treatment and surgery. Kadanka et al ${ }^{4,19}$ proposed that $80 \%$ of mild CSM patients will have better recovery, with or without surgery. Ten years of prospective randomized controlled trials showed that for mild-to-moderate CSM patients, no obvious significant difference exists between conservative treatment and surgery. For both groups, patient conditions all improve at first, but then grow worse. ${ }^{20}$ Shimomura et al. ${ }^{5}$ proposed that $80 \%$ of mild CSM patients are in a continuous stable state during 3 years of follow-up. Epstein et al..$^{21}$ and Sampath et al..$^{22}$ also supported this conclusion. Yarbrough et al. ${ }^{23}$ pointed out that as time goes on, individual subjective feeling and health status may decrease, which may affect the individual choice for therapy method.

Traditional concepts suggested that CSM is an inert disease, because of the continual deterioration of spinal cord function caused by internal compression of osteophyte. Therefore, decompressing surgery can prevent disease development, while conservative treatment effects should not be obvious, because it cannot decompress the spinal cord. However, Matz ${ }^{24}$ proposed that the pathogenesis of CSM consists of some dynamic factors other than statistic compression. Once more obvious clinical symptoms appear, no matter what measures are taken, the disease will still persistently advance. By this assumption, conservative treatment can prevent the progress of disease and improve symptoms, especially for mild CSM, but this conclusion is not convincing, because the research samples are not large enough. However, researchers had to admit that conservative treatment is important in the early stage of the disease, while surgery may not achieve the desired clinical effects through a long-time follow-up.

Some limitations exist with this, as with many previous studies. Because of occult onset at the early stage of CSM, the clinical manifestation is easily ignored. The diagnosis of mild CSM is usually made according to the JOA score, while the JOA score is a patient's subjective assessment scale, and may not objectively and accurately reflect the disease situation. The clinical symptoms may not be in accord with the imaging manifestation; for example, clinical symptoms being relatively mild, while MRI shows severe compression of the spinal cord, and even intramedullary ISI on T2WI. Oshima and group $^{6}$ published research to clarify mild CSM, especially the natural course and prognostic factors of those with intramedullary ISI. He retrospectively studied 45 CSM patients not having surgery at first visit, some of whom turned to surgery when they showed neurologic deterioration. He further compared the relative prognostic factors of these kinds of patients and the other patients still insisting on conservative treatment. The results showed that, during 5 and 10 years after the initial diagnosis, 82 and $56 \%$ of patients still did not need surgery. Large range of motion, segmental kyphosis and instability at the narrowest canal were risk factors for converting to surgery in the future.

In analysis of previous research, we filtered the patients' literatures, and the index of intramedullary ISI on MRI T2WI was analyzed, to increase the reliability of the findings. Patients with shorter disease course and larger segmental lordosis have better clinical outcomes, which means mild CSM patients with intramedullary ISI on MRI T2WI need therapeutic intervention as early as possible, whatever kind of therapy is chosen. For patients who have segmental kyphosis at the most compressed segment, any therapy may not be helpful. Although the reason why the existence of segmental kyphosis influenced the prognosis remains uncertain, long-standing segmental malalignment might have caused chronic ischemia of the spinal cord. ${ }^{25,26}$

In conclusion, for mild CSM patients with intramedullary ISI on MRI T2WI, no significant differences between surgery and conservative treatment were found during the short-term follow-up. However, the clinical effects of patients with shorter disease course and larger segmental lordosis are better than that with longer course and segmental kyphosis.

This study has several limitations. First, it is a retrospective study. Second, some limitations exist, such as short follow-up periods and economic conditions that may affect the therapy chosen. For mild CSM patients with intramedullary ISI, natural course and surgery time must be further analyzed in randomized controlled trials.

\section{DATA ARCHIVING}

There were no data to deposit.

\section{CONFLICT OF INTEREST}

The authors declare no conflict of interest.

\section{ACKNOWLEDGEMENTS}

This study was supported by the 1255 Project of Changhai Hospital of Shanghai (Number: CH125520900, CH125540200).

1 Tracy JA, Bartleson JD. Cervical spondylotic myelopathy. Neurologist 2010; 16 $176-187$

2 Takahashi M, Yamashita Y, Sakamoto Y, Kojima R. Chronic cervical cord compression: clinical significance of increased signal intensity on MR images. Radiology 1989; 173: 219-224.

3 Li F, Chen Z, Zhang F, Shen H, Hou T. A meta-analysis showing that high signal intensity on T2-weighted MRI is associated with poor prognosis for patients with cervical spondylotic myelopathy. J Clin Neurosci 2011; 18: 1592-1595.

4 Kadanka Z, Mares M, Bednarik J, Smrcka V, Krbec M, Chaloupka R et al. Predictive factors for mild forms of spondylotic cervical myelopathy treated conservatively or surgically. Eur J Neurol 2005; 12: 16-24.

5 Shimomura T, Sumi M, Nishida K, Maeno K, Tadokoro K, Miyamoto $\mathrm{H}$ et al. Prognostic factors for deterioration of patients with cervical spondylotic myelopathy after nonsurgical treatment. Spine (Phila Pa 1976) 2007; 32: 2474-2479.

6 Oshima Y, Seichi A, Takeshita K, Chikuda H, Ono T, Baba S et al. Natural course and prognostic factors in patients with mild cervical spondylotic myelopathy with increased signal intensity on T2-weighted magnetic resonance imaging. Spine (Phila Pa 1976) 2012; 37: 1909-1913. 
7 Vedantam A, Jonathan A, Rajshekhar V. Association of magnetic resonance imaging signal changes and outcome prediction after surgery for cervical spondylotic myelopathy. J Neurosurg Spine 2011; 15: 660-666.

8 Matsuda Y, Miyazaki K, Tada K, Yasuda A, Nakayama T, Murakami H et al. Increased MR signal intensity due to cervical myelopathy. Analysis of 29 surgical cases. J Neurosurg 1991; 74: 887-892.

9 Wada E, Ohmura M, Yonenobu K. Intramedullary changes of the spinal cord in cervical spondylotic myelopathy. Spine 1995; 20: 2226-2232.

10 Morio Y, Yamamoto K, Kuranobu K, Murata M, Tuda K. Does increased signal intensity of the spinal cord on MR images due to cervical myelopathy predict prognosis? Arch Orthop Trauma Surg 1994; 113: 254-259.

11 Chen CJ, Lyu RK, Lee ST, Wong YC, Wang LJ. Intramedullary high signal intensity on T2-weighted MR images in cervical spondylotic myelopathy: prediction of prognosis with type of intensity. Radiology 2001; 221: 789-794.

12 Fernandez de Rota JJ, Meschian S, Fernandez de Rota A, Urbano V, Baron M. Cervical spondylotic myelopathy due to chronic compression: the role of signal intensity changes in magnetic resonance images. J Neurosurg Spine 2007; 6: 17-22.

13 Naderi S, Ozgen S, Pamir MN, Ozek MM, Erzen C. Cervical spondylotic myelopathy: surgical results and factors affecting prognosis. Neurosurgery 1998; 43: 43-49.

14 Ramanauskas WL, Wilner HI, Metes JJ, Lazo A, Kelly JK. MR imaging of compressive myelomalacia. J Comput Assist Tomogr 1989; 13: 399-404.

15 Ito T, Oyanagi K, Takahashi H, Takahashi HE, Ikuta F. Cervical spondylotic myelopathy: clinicopathologic study on the progression pattern and thin myelinated fibers of the lesions of seven patients examined during complete autopsy. Spine 1996; 21: 827-833.

16 Yone K, Sakou T, Yanase M, ljiri K. Preoperative and postoperative magnetic resonance image evaluations of the spinal cord in cervical myelopathy. Spine (Phila Pa 1976) 1992; 17 (10 Suppl), S388-S392.
17 Ohshio I, Hatayama A, Kaneda K, Takahara M, Nagashima K. Correlation between histopathologic features and magnetic resonance images of spinal cord lesions. Spine 1993; 18: 1140-1149.

18 Vedantam A, Rajshekhar V. Does the type of T2 weighted hyperintensity influence surgical outcome in patients with cervical spondylotic myelopathy? Eur Spine J 2013; 22: 96-106.

19 Kadanka Z, Mares M, Bednanik J, Smrcka V, Krbec M, Stejskal L et al. Approaches to spondylotic cervical myelopathy: conservative versus surgical results in a 3-year followup study. Spine (Phila Pa 1976) 2002; 27: 2205-2210.

20 Kadanka Z, Bednarik J, Novotny O, Urbánek I, Dušek L. Cervical spondylotic myelopathy: conservative versus surgical treatment after 10 years. Eur Spine $J$ 2011; 20: 1533-1538.

21 Epstein JA, Janin Y, Carras R, Lavine LS. A comparative study of the treatment of cervical spondylotic myeloradiculopathy. Experience with 50 cases treated by means of extensive laminectomy, foraminotomy, and excision of osteophytes during the past 10 years. Acta Neurochir (Wien) 1982; 61: 89-104.

22 Sampath $\mathrm{P}$, Bendebba M, Davis JD, Ducker TB. Outcome of patients treated for cervical myelopathy. A prospective, multicenter study with independent clinical review. Spine (Phila Pa 1976) 2000; 25: 670-676.

23 Yarbrough CK, Murphy RK, Ray WZ, Stewart TJ. The natural history and clinical presentation of cervical spondylotic myelopathy. Adv Orthop 2012; 2012: 480643.

24 Matz PG. Does nonoperative management play a role in the treatment of cervical spondylotic myelopathy? Spine J 2006; 6 (6 Suppl), 175S-181S

25 Albert TJ, Vacarro A. Postlaminectomy kyphosis. Spine (Phila Pa 1976) 1998; 23. 2738-2745.

26 Uchida K, Nakajima H, Sato R, Yayama T, Mwaka ES, Kobayashi S et al. Cervical spondylotic myelopathy associated with kyphosis or sagittal sigmoid alignment: outcome after anterior or posterior decompression. J Neurosurg Spine 2009; 11: 521-528. 